\title{
miR-30a-5p inhibits osteogenesis and promotes periodontitis by targeting Runx2
}

\author{
Xiangdong Liu' ${ }^{1 \dagger}$, Bo Yang ${ }^{2,3 \dagger}$, Yan Zhang ${ }^{2,4 \dagger}$, Xiaorui Guo ${ }^{2,5}$, Qianjuan Yang ${ }^{2}$, Xiaojing Liu², Qingxia Bai ${ }^{2}$ and
} Qun Lu $2^{2^{*}}$

\begin{abstract}
Background: Periodontitis is the most extensive chronic inflammatory bone resorption disease. MiRNAs offer a potential way for potential therapy. Indeed, miR-30a-5p had an increasing expression in periodontitis gingivae, but whether it promotes osteogenesis and inhibits inflammation remains unknown.
\end{abstract}

Methods: Periodontitis model was exhibited by wire ligation and verified by micro-CT and HE staining; qPCR was used to detect the expression of miR-30a-5p; miR-30a-5p inhibitors and mimics were transfected into MC3T3-E1 cell line by lipofectamine 3000; The dual luciferase reporter gene experiment and RIP experiment were used to detect the relationship between miR-30a-5p and Runx2; Rescue experiment was used to verify the relationship between miR30a-5p and Runx2.

Results: Periodontitis model was exhibited successfully and miR-30a-5p was overexpressed at the bone and gingival tissues of this model. miR-30a-5p inhibitors not only promoted the osteogenesis but also relieved inflammation. Runx2 is a target of miR-30a-5p by dual luciferase reporter gene experiment and RIP experiment. Rescue experiments revealed that miR-30a-5p inhibitors would promote osteogenesis and relieve inflammation by targeting Run $\times 2$ in inflammation of MC3T3-E1 cell line.

Conclusions: That all suggested that miR-30a-5p-mediated-Runx2 provided a novel understanding of mechanism of periodontitis.

Keywords: Periodontitis, miRNA, Runx2, Rescue

\section{Background}

Periodontitis, which accompanied with pathologic loss of soft and hard tissue [1], is related to the mutual effects between host immunity and dysfunctional oral microbiota located in the subgingival niche [2-4]. In developing countries, periodontitis is more prevalent than developed countries [5]. Uncontrolled periodontitis

\footnotetext{
*Correspondence: luqun22@hotmail.com

${ }^{\dagger}$ Xiangdong Liu, Bo Yang and Yan Zhang contributed equally to this work.

2 State Key Laboratory of Military Stomatology and National Clinical Research Center for Oral Diseases and Shaanxi Key Laboratory of Oral Diseases, Department of Operative Dentistry and Endodontics, School of Stomatology, The Fourth Military Medical University, Xi'an, China Full list of author information is available at the end of the article
}

may cause teeth loss, diabetes mellitus [6], preterm low birthweight [7] and cardiovascular disease [8], so it is important to intervene it early [9]. MicroRNA (miRNA) is a non-coding RNA and plays the role of silencing the target genes through complementary base pairing [10, 11]. Several serum miRNAs including miR-207, miR495, miR-376b-3p may be the biomarkers of periodontitis [12]. A research by miRNA microarray showed that miR-30a-5p increased in periodontitis [13]. Furthermore, there are also many different miRNAs involved in the process and pathology of periodontitis [14, 15]. MiR21 has been reported to inhibit the inflammation and down-regulate in periodontitis [16]. miR-155-5p/SIRT1 regulatory network alleviated inflammatory of PDLSCs 
[17]. MiR-30a-5p, which located in chromosome 6:71, involved aspects of diseases and physiology. miR-30a-5p combined miR-769-59 could be a biomarker from plasma for diagnosis in in oral squamous cell carcinoma (OSCC) [18]. But whether miR-30a-5p influenced the biological functions of periodontitis remains unknown.

Runx2 (the osteoblast transcription factor 2) is a transcription factor for osteoblast differentiation [19]. Komori et al. [20] found that osteoblasts were almost absent in Runx $2^{-1-}$ mice. Meanwhile, it plays a vital role in chondrocyte maturation [21]. For special deletion of Runx $2^{-1-}$ of skeleton, chondrocyte maturation was severely inhibited [22]. Overexpression of Runx2 accelerated progression of post-traumatic osteoarthritis in adult mice [23]. Runx2 improved osteogenesis of bone marrow mesenchymal stem cells (BMSCs) in vitro and repaired cartilage repair in vivo [24]. A recent study indicated that rs59983488 in Runx2 was associated with persistent apical periodontitis (PAP) for a higher risk of developing PAP [25]. However, whether miR-30a-5p targeting Runx2 influences the process of periodontitis remains unknown.

In this study, we designed the animal model of periodontitis and aimed to investigate the expression of miR-30a-5p and its effects on the osteogenesis and inflammation by targeting Runx 2 in periodontitis.

\section{Methods}

\section{Ethics statement}

All experiments performed in this study were confirmed by the Ethical Committee of the School of Stomatology, The Fourth Military Medical University (Xi'an, China, NO: kq-013) and in compliance with ARRIVE guidelines (https://arriveguidelines.org). All methods were accordance with relevant guidelines including the revised Animals (Scientific Procedures) Act 1986 in the UK and Directive 2010/63/EU in Europe. All lab work and methods were performed by the authors of this paper.

\section{Animal model}

32 SD male rats $(250 \mathrm{~g} \pm 10 \mathrm{~g})$ in the Experimental Animal Center of the School of Stomatology, The Fourth Military Medical University were selected and adaptive fed in a SPF (Specific Pathogen Free) condition with temperature of $21-23^{\circ}$, relative humidity of $30-60 \%$ and $12 \mathrm{~h}$ lighting/dark alternating environment.

Periodontitis models built by the ligature-induced method, which has been widely used in the previous studies [4]. Firstly, the rats were anesthetized by intraperitoneal injecting $5 \mathrm{mg} / \mathrm{kg}$ xylazine (Albrechts $\mathrm{GmbH}$, Aulendorf, Germany) and $85 \mathrm{mg} / \mathrm{kg}$ ketamine (Albrechts $\mathrm{GmbH}$, Aulendorf, Germany) into the abdominal cavity to anesthetize them, then the periodontal tissue of the maxillary 1st molar were separated and rooted with
$0.2 \mathrm{~mm}$ orthodontic ligation wire, periodontal tissue on the right was used as control group. Rats were sacrificed by overdose anesthesia and organs were collected for pathological examination.

\section{Micro-computed tomography (micro-CT)}

Maxillary bone specimens were dissected at 0,7 days and 21 days after ligation and fixed into $4 \%$ paraformaldehyde, respectively. Samples were analyzed of microstructures of bone loss by the micro-CT system (Siemens, Inveon, Germany) in the maxillary area, which were scanned at parameters of $80 \mathrm{kV}$ and $80 \mu \mathrm{A}$ with a pixel size of $18 \mu \mathrm{m}$ and an exposure time of $3000 \mathrm{~ms}$. GEHC Micro View software was used to reconstruct the 3D images after scanning.

\section{HE and TRAP staining}

After micro-CT scanning, the specimens were decalcified in 17\% EDTA solution (Thermo scientific, Waltham, MA USA) for 30-40 days. After dehydration, paraffin embedding was performed, and $5 \mu \mathrm{m}$ continuous sections were made and nearly $5 \mu \mathrm{m}$ continuous sections were made. HE and TRAP staining were performed on the sections at three locations according to the manufacturer's protocols.

\section{Cell culture and LPS inducing}

MC3T3-E1 and $293 \mathrm{~T}$ cell lines were obtained from ATCC (ATCC, Manassas, VA, USA). All cell lines were cultured in RPMI-1640 (Sigma) with 10\% FBS (Sigma), $1 \% 100$ units $/ \mathrm{ml}$ penicillin and $100 \mu \mathrm{g} / \mathrm{ml}$ streptomycin (Sigma) in an incubator $\left(37^{\circ} \mathrm{C}, 5 \% \mathrm{CO}_{2}\right.$ ) (NUAIRE, Plymouth, MN, USA). For simulation of periodontitis in vivo, $1 \mathrm{ug} / \mathrm{ml}$ lipopolysaccharide (LPS) from Porphyromonas gingivalis were added at serum-free medium of MC3T3E1 cell line.

MiRNA-30a-5p mimic/inhibitor and si-Runx2 transfection MC3T3-E1 cell line was plated into 6-wells plate after transfected with $1.2 \times 10^{5}$ cells $/ \mathrm{cm}^{3}$. After $24 \mathrm{~h}$, cells were washed by $1 \times$ PBS (Sigma) three times and configured with transfection solution (10ul opti-Mem medium $+0.2 \mathrm{ul}$ lipofectamine 3000 (Promega, Madison, Wisconsin, USA) was prepared with liquid A; 10 ul Medium + 100 nM MiR-30a-5p mimics, inhibitor, siRunx2/NC solution were liquid B) (Genepharm, Suzhou, China). miR-30a-5p mimics, inhibitor and si-Runx2 were designed from Sangon Biotech company (Sangon Biotech, Shanghai, China). The sequences have been shown in Table 1. After preparation, Solution A and Solution B were mixed for $15 \mathrm{~min}$ at $1: 1$ ratio by volume at a room temperature, and the liposome complex was added to the cellular pore plate, supplemented with serum-free 
Table 1 Sequencing of primers, mimics, inhibitors and siRunx2

\begin{tabular}{lll}
\hline Name & Forward & Reverse \\
\hline miR-30a-5p & UGUAAACAUCCUCGACUGGAAG & CAGTGCGTGTCGTGGAGT \\
IL1-R1 & CTGCTGTCGCTGGAGATTGAC & TTGGCAGGTACAAACCAAAGAT \\
IL1-R2 & GTTTCTGCTTTCACCACTCCA & GAGTCCAATTACTCCAGGTCAG \\
TNFR1 & GGGGATACATCCATCAGGGGT & GCTCGGACAGTCACTCACC \\
ALP & CAGCGGGTAGGAAGCAGTTTC & CCCTGCACCTCATCCCTGA \\
Runx2 & GACTGTGGTTACCGTCATGGC & ACTTGGTTTTCATAACAGCGGA \\
OC & CCCATCTTTGAGCATCTTGGT & GCCCAGCCTGAGTAGTGAAG \\
CoL1 & AATGGAAGTTCTACTCGCGTAGG & TTCTCGCCTGGTTGACCTTTG \\
GAPDH & AATGGATTTGGACGCATTGGT & TTTGCACTGGTACGTGTTGAT \\
miR-30a-5p mimic & UCCAGUCGAGGAUGUUUACAUU \\
mimic NC & UGUAAACAUCCUCGACUGGAAG & ACGUGACACGUUCGGAGAATT \\
Inhibitor NC & UUCUCCGAACGUGUCACGUTT & ACGUGACACGUUCGGAGAATT \\
Si-Runx2-492 & UUCUCCGAACGUGUCACGUTT & UAGUUCUCAUCAUUCCCGGTT \\
Si-Runx2-909 & CCGGGAAUGAUGAGAACUATT & UAAGACUGGUCAUAGGACCTT \\
Si-Runx2-1059 & GGUCCUAUGACCAGUCUUATT & AUUUGGAUUUAUAGCGUGTT \\
U6 & CACGCUAUUAAAUCCAAAUTT & TTCTCGGCGTCTTCTTTCTCG \\
\hline
\end{tabular}

medium, and then incubated them in an incubator for $24 \mathrm{~h}$.

\section{Osteogenesis differentiation induction}

Cells were cultured into 6-wells plate for $24 \mathrm{~h}$ and then were transfected miR-30a-5p mimics, inhibitor, si-Runx2, or si-Runx2 plus miR-30a-5p inhibitor and negative controls. Osteogenic induction solution $(10 \mathrm{mmol} / \mathrm{L}$ sodium glycerophosphate, $50 \mathrm{~g} / \mathrm{mL}$ ascorbic acid, $10 \mathrm{~mol} / \mathrm{L}$ dexamethasone and $100 \mathrm{ml} / \mathrm{L}$ fetal bovine medium) was replaced $4 \mathrm{~h}$ and cells were cultured in a $\mathrm{CO}_{2}$ incubator.

\section{Alkaline phosphatase and Alizarin red staining}

After osteogenic induction for 7 or 21 days, cell line was fixed with $4 \%$ paraformaldehyde (Sigma) for $30 \mathrm{~min}$ at a room temperature, and then washed 3-5 times with $1 \times$ PBS for 3-5 min; Secondly, BCIP/NBT staining solution (Cyagen, Santa Clara, CA) or Alizarin red (Solarbio) were added to the cells and incubated at a room temperature for 5-30 min in the dark. Until the expected color appeared, BCIP/NBT or Alizarin red dye solution (Cyagen) were removed and washed 2-3 times with distilled water and taken pictures.

\section{Dual-luciferase reporter gene}

To verify the targeting relationship between miR-30a-5p and Runx2, we constructed a dual-luciferase reporter vector (WT) and a mutant dual-luciferase reporter vector (MUT), respectively. Sequencing results were shown in the Additional file 1: Figure S1A-1B. miR-30a-5p mimic, WT and MUT were co-transfected into 293T cell line for $48 \mathrm{~h}$. The luciferase activity measured using a Dual-Glo Luciferase Assay System and assayed with a Multimode Plate Reader with a normalization of Renin luciferase activity.

\section{RNA extraction and quantitative real-time polymerase chain reaction (qRT-PCR)}

RNA from the cell line extracted from Trizol reagent according to the manufacturer's protocols. A Nano-Drop spectrophotometer was used to measure the concentration of RNA; mRNA reversed into cDNA by Takara Prime Script RT reagent Kit with gDNA Remover, and miRNA reversed by using All-in-One miRNA qRT-PCR System according to the manufacturer's protocols using the comparative CT $(\Delta \Delta \mathrm{Ct})$ method. The primer information was shown in Table 1.

\section{RNA immunoprecipitation (RIP)}

RIP was used to detect endogenous interaction between miR-30a-5p and Runx2. Firstly, cells were transfected into miR-30a-5p mimics and negative control, respectively. Cell lysates and magnetic beads were together incubated with anti-Argonaute2 (antibodies-online, Aachen, Germany) and eluted absolutely. The precipitated RNA was analyzed by qRT-PCR with an input as positive control and IgG as a negative control (Millipore, Darmstadt, Germany).

\section{Statistical analysis}

Mean \pm Standard Error of Mean (mean \pm SEM) methods were used for all data, Student's $t$ test was carried out for statistical analysis using GraphPad Prism 9 (SPSS v.19.0, 
IBM, Chicago, IL, USA). Values of $P<0.05$ were considered statistically significant.

\section{Results}

miR-30a-5p was up-regulated in gingiva tissues and bones in periodontitis of rat model

For detecting the expression of miR-30a-5p, a rat model of periodontitis was exhibited by ligature-inducing. As micro-CT results showed in Fig. 1A, bone loss occurred after ligation-inducing for 7 days and a deeper loss in 21 days. HE and TRAP results indicated that inflammation cytokines and osteoclast cells appeared after ligation at 7 days and reached a higher expression level at 21 days (Fig. 1B, C). miR-30a-5p was upregulated in bone and gingiva tissues comparing with normal ones (Fig. 1D, E). It suggested that miR-30a-5p involved in the occurrence and development of periodontitis.

\section{miR-30a-5p was upregulated in MC3T3-E1 cell line after LPS inducing}

MC3T3-E1 cell line were cultured (Fig. 2A) and oil red staining result showed its adipogenic tendency (Fig. 2B). Results of alkaline phosphatase (ALP) staining (Fig. 2C) and qPCR of osteogenic genes (Fig. 2D) showed its osteogenic differentiation. qRCR results showed that miR30a-5p was upregulated in MC3T3-E1 cell line after LPS-inducing treatment (Fig. 2E). A much more increasing level could be seen in 1, 4, 7, 14 days with LPS-inducing (Fig. 2F).

\section{miR-30a-5p inhibitor reduced the expression} of inflammatory factors and promoted the expression of osteogenesis factors

As showed in Fig. 3A, B, miR-30a-5p was upregulated in the mRNA level when transfecting miR-30a-5p mimics while downregulated in miR-30a-5p inhibitor comparing to relative control groups. The inflammatory factors IL1R1, IL1-R2 and TNFR1 were upregulated when transfecting miR-30a-5p mimics (Fig. 3C), accompanied by downregulated transfecting miR-30a-5p inhibitors (Fig. 3D). MiR-30a-5p inhibitors promoted osteogenesis match along with miR-30a-5p mimics inhibited osteogenesis, based on results of Alkaline phosphatase and alizarin red staining (Fig. 3E, F). Osteogenic factors ALP, Runx2, OC, and Col-1 were upregulated when transfected miR-30a-5p inhibitor (Fig. 3G), accompanying by downregulated when transfected miR-30a-5p mimics (Fig. 3H), based on the result of qRT-PCR. These indicated that miR-30a-5p involved in regulation of osteogenesis and inflammation in periodontitis, and miR-30a-5p inhibitors relieved inflammation and promoted the osteogenesis.

\section{Runx2 is a target of miR-30a-5p}

The dual-luciferase report gene experiment is a direct method to verify whether the target genes are the target of miRNAs. The binding sites of miR-30a-5p and Runx2 were shown in Fig. 4A, and the dual-luciferase report gene results showed that Runx 2 is a target of miR-30a-5p (Fig. 4B). The results showed that Runx 2 increased in the mRNA level when transfecting miR-30a-5p mimics and declined with miR-30a-5p inhibitors (Fig. 4C). RIP experiment showed that miR-30a-5p and Runx2 assembled into Argonaute- 2 protein, IgG was a negative control and input was a positive control (Fig. 4D, E), while miR-30-5p couldn't interact with ALP, OC, Col1 and then not assembled into Argonaute- 2 protein (Fig. $4 \mathrm{~F}-\mathrm{H}$ ). The results indicated that the action of miR-30a-5p in reducing the expression of ALP, OC and Col1 genes were indirect.

\section{Co-transfecting si-Runx2 and miR-30a-5p inhibitor combatted the effects of osteogenesis and inflammatory of si-Runx2 in MC3T3-E1 cell line by rescue experiments} qRT-PCR result showed that si-Runx2-909 silenced the expression of Runx2 efficiently (Fig. 5A), so it was utilized for following experiments and named si-Runx2. Inflammatory factors IL1R-1, IL1R-2 and TNFR upregulated when transfecting si-Runx2, however si-Runx2 plus miR-30a-5p inhibitors combatted the promoted effect (Fig. 5B). Osteogenesis factors Runx2, ALP, OC, COL1 (Fig. 5C, D) were downregulated when transfecting siRunx2, however si-Runx2 plus miR-30a-5p inhibitors combatted inhibitory effect. Result of Alkaline phosphatase staining and alizarin red also indicated si-Runx2 plus miR-30a-5p inhibitor combatted the inhibitory osteogenesis effect of si-Runx2 (Fig. 5E, F).

\section{Discussion}

Periodontitis, one of the multifactorial infectious diseases, is characterized by gingival inflammation and bone loss $[14,26]$. Modern molecular technologies including pathogenic agents, haplotype aberrations and gene therapy have been used friendly in recent years [1]. For instance, CXCL12 (the chemokine stromal-cell derived factor-1) was increased in human periodontitis by targeting CXCR4. So, seeking a gene for inhibit inflammation and relieve bone loss makes it senses.

MiRNAs are evolutionarily conserved non-coding RNA molecules (20-22 nucleotides in length) that primarily function to prevent mRNA translation or initiate mRNA degradation at the posttranscriptional level via binding to the 3' untranslated regions (3' UTR) of their target mRNAs [27]. Besides, some animal miRNAs may also target 5'UTR and coding regions according to bioinformatics predictions and others experiments [28]. 
A
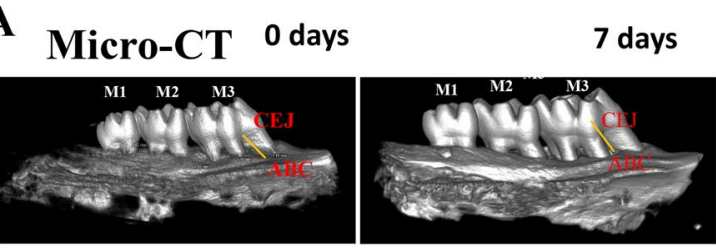

days

21 days
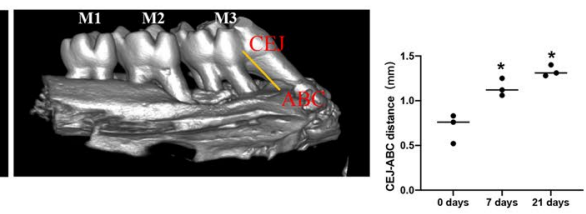

0 days

7 days

21 days
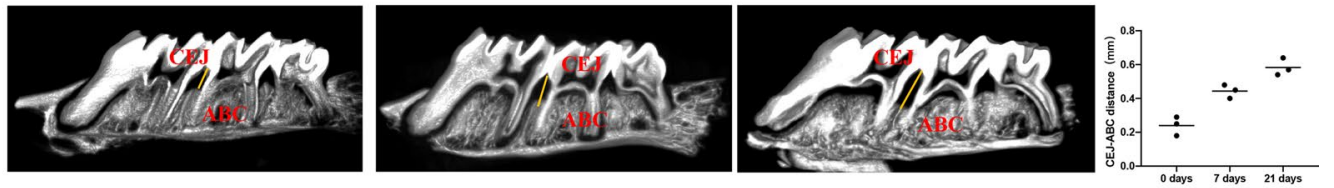

B
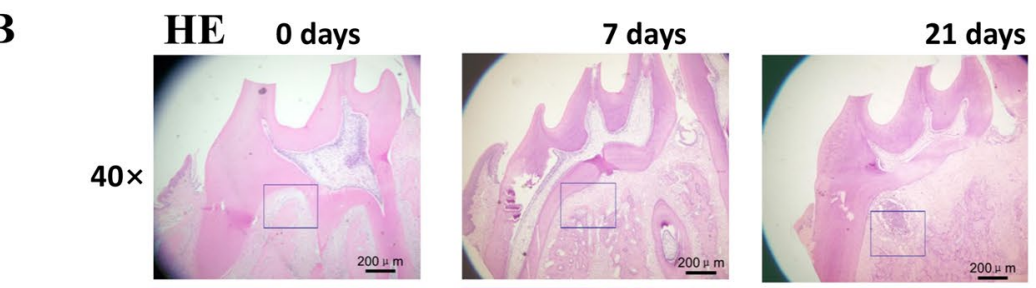

21 days
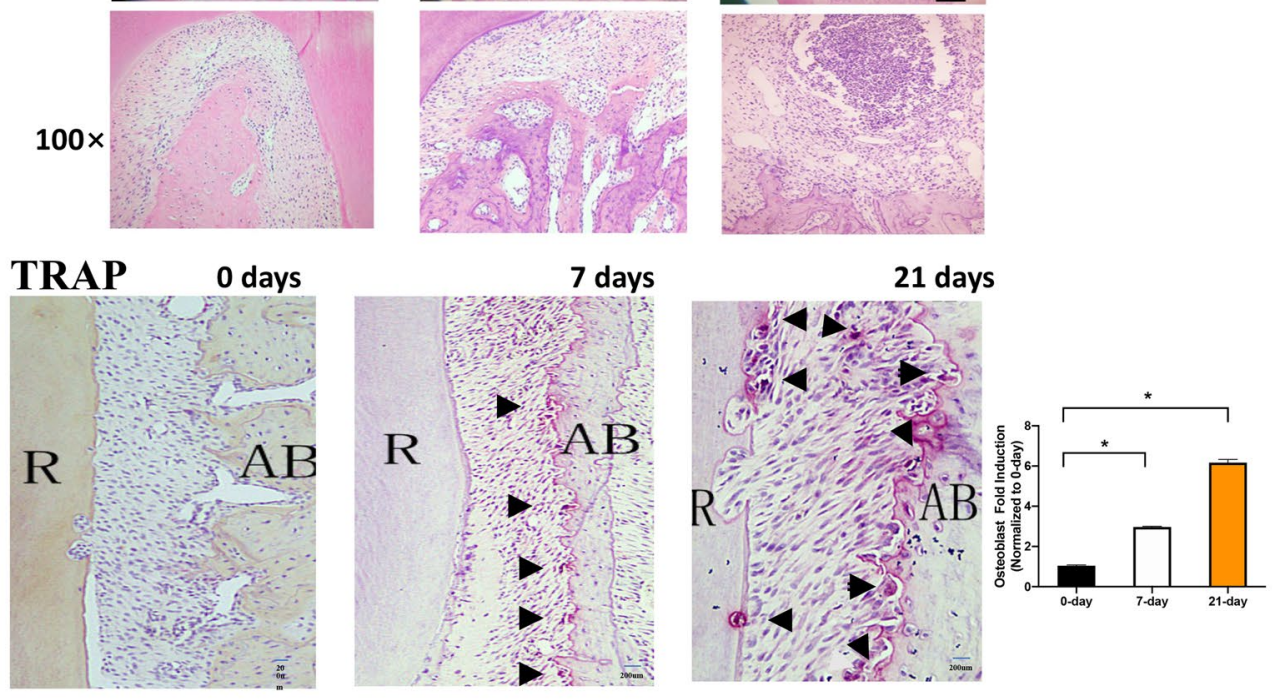

D

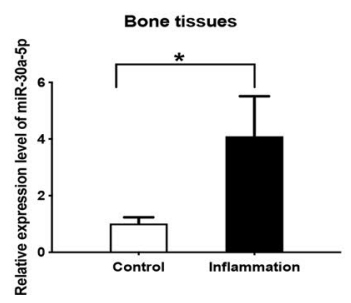

E

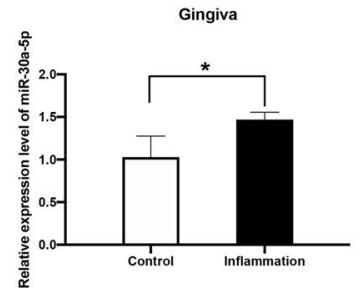

Fig. 1 miR-30a-5p was upregulated at bone and gingiva in periodontitis rat model. A Bone loss was examined by micro-CT after ligation for 7 , 21 days. B Cellular morphology was examined by HE after ligation for 0, 7, 21 days. C Osteoclasts were examined by TRAP after ligation for 0, 7, 21 days. D The expression of miR-30a-5p was examined by qPCR of bone tissues from periodontitis (inflammation) and healthy controls (control). $\mathbf{E}$ The expression of miR-30a-5p was examined by qPCR of gingival tissues from periodontitis (inflammation) and healthy controls (control). (R: root; $\mathrm{AB}$ : alveolar bone). All data are shown as mean $\pm \mathrm{SEM} .{ }^{*} p<0.05 ;{ }^{* *} p<0.01$ 


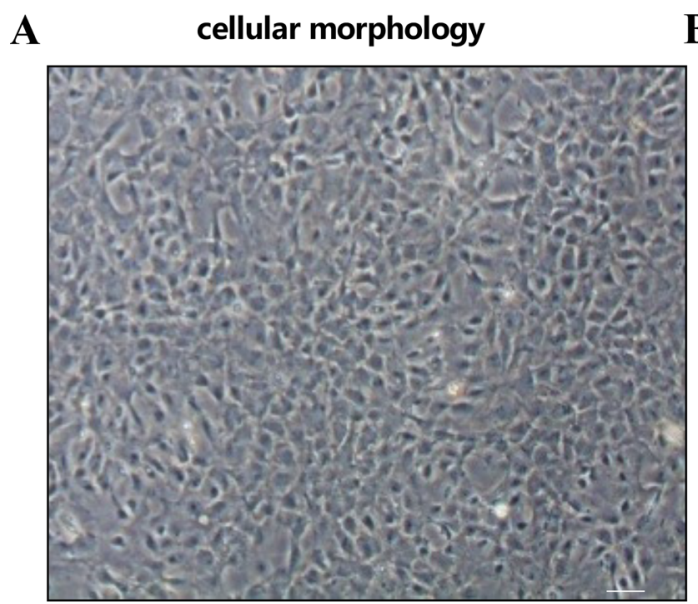

B Adipogenesis

C

Osteogenesis

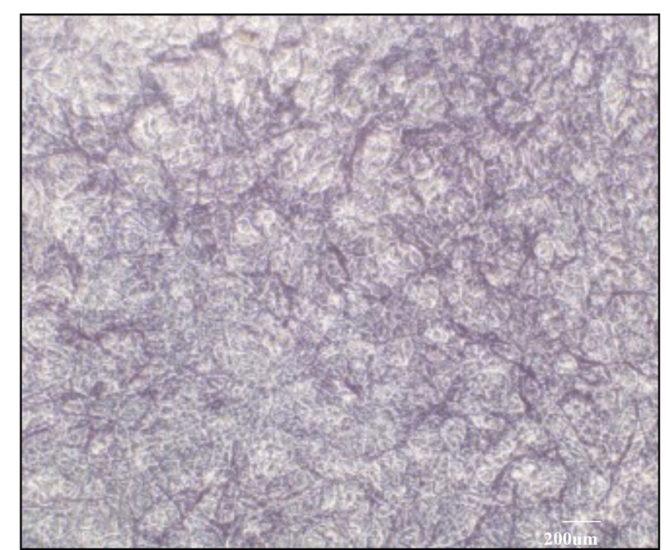

D

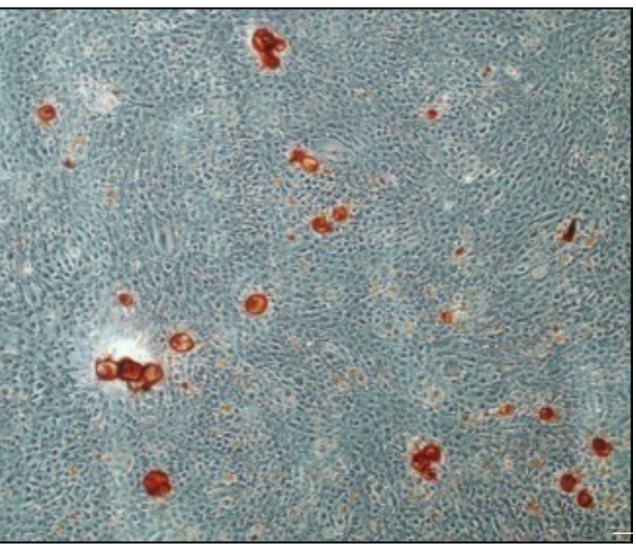

$\square$ Control

- Osteogenesis

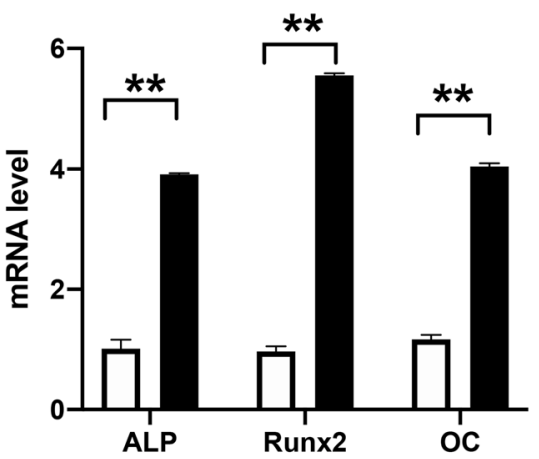

E

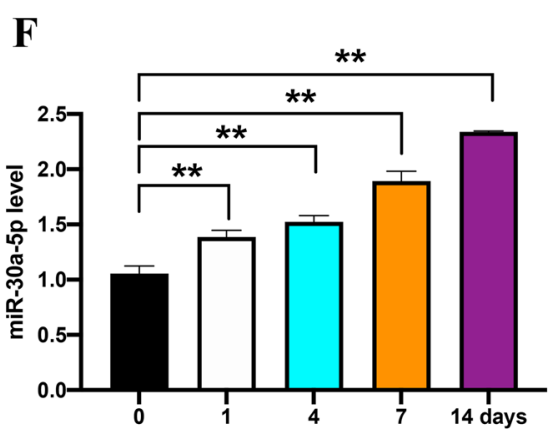

Fig. 2 miR-30a-5p was upregulated in MC3TC-E1 cell line under LPS-inducing. A Microscopic image of P3 of MC3T3-E1 taken by microscope. B The level of lipid droplet was examined by oil-red staining in MC3T3-E1 cells after 14 days after adipogenic induing. C The level of ALP was examined by ALP staining in MC3T3-E1 cells after 14 days after osteogenic induing. D The expression of ALP, Runx 2 and OC were examined by qPCR in MC3T3-E1 cells during osteogenic induing. E The expression of miR-30a-5p was examined by qPCR in MC3T3-E1 cells after LPS inducing (1 ug/ml). F The expression of miR-30a-5 $p$ was examined by qPCR in MC3T3-E1 cells after LPS induing (1 ug/ml) for 0, 1, 4, 7, 14 days. All data are shown as mean \pm SEM. ${ }^{*} p<0.05 ;{ }^{* *} p<0.01$

Mounting evidence showed that miRNAs involved in periodontal diseases including periodontitis recently [14, 29]. Lee et al. found six miRNAs were upregulated not only in inflammation but also in bone loss and injured early in 2001. miR-30a-5p, one of six miRNAs, which located in Chromosome 1: 23,311,350-23,311,420, was identified an amplified expression in periodontitis than healthy ones [13]. Furthermore, miR-30a-5p was a 
prognostic biomarker in colorectal cancer [30], a suppressor gene of non-small cell lung cancer [31]. miR$30 a-5 p$ was found to be relationship with allergic disease [32]. A recent study showed that miR-30a-5p, the mature product of miR-30a, promoted to exacerbate inflammation and organ damage in sepsis model [33].

In this study, miR-30a-5p was found to have an increasing expression in gingiva and bone tissues of the periodontitis rat model, comparing with that of normal periodontal tissue in vivo. In vitro, miR-30a-5p was equivalent upregulated in MC3T3-E1 cell line as time extension after LPS-inducing and reach at a higher level in 14 days testing by qRT-PCR. MiR-30a-5p were upregulated not only in soft tissue but also in hard tissue of periodontitis. All suggest that miR-30a-5p involved in process of periodontitis.

miR-30a-5p mimics, inhibitors were designed to transfect MC3T3 cell line. miR-30a-5p inhibitors promoted expression of the osteogenesis factors Runx2, ALP, OC, COL1 and reduced expression of inflammation factors IL1R-1, IL1R-2, TNFR, accompany with miR-30a-5p mimics played reversed roles, which suggested miR$30 a-5 p$ played a regulatory role in the osteogenesis and inflammation of periodontitis.

miRNAs modulate gene expression at the post-transcriptional level either by promoting mRNA degradation or by inhibiting messenger RNA (mRNA) translation [34]. According to the Target Scan websites (http://www. targetscan.org/mamm_31/), we found more than 1576 target genes of miR-30a-5p including Runx2 partly because it has a considerably long 3'-UTR $(\sim 4 \mathrm{~kb})$. Runx2 is not only an inducer of chondrocyte and osteoblast differentiation but also a gene of mesenchymal precursor cell differentiation [21, 35]. The expression of Runx2 is distinct in different kinds of cells. For instance, Runx2 weakly expressed in uncommitted mesenchymal cells, highly in pre-osteoblasts, reached the maximal level in immature osteoblasts, and then down-regulated in mature osteoblasts [36]. Overexpression of Runx2 accelerated osteoblast differentiation under the regulation of the Prrx1 promoter [37, 38]. Additionally, microRNA-218 decreased the expression of inflammatory cytokines in acute lung injury model targeting Runx2 recent reports by Zhou et al. [32]. Therefore, Runx 2 might be related to the inflammation. That is a potential reason for miR30a-5p inhibitors promoting osteogenesis but inhibiting inflammation through the overexpression of Runx2. MiR-30a-5p alleviated the LPS-inducing inflammation by targeting Runx2 in A549 cell line [39]. Evidence whether miR-30a-5p plays a role in osteogenesis and inflammation by targeting Runx2 in MC3TC-E1 cell line need to be further explored.

We confirmed that Runx 2 was a target gene of miR$30 a-5 p$ with certain binding sites by the dual luciferase gene reporter experiments. RIP is a direct method to detect the relationship between two different genes [40]. In this study, the expression of Argonaute-2 protein which directly link miR-30a-5p and Runx2 increased after transfecting miR-30a-5p mimics by RIP. RIP experiments also indicated that these are not direct conjunction between miR-30a-5p and ALP, OC, COL1. As shown in Fig. 5D ALP, OC and Col1 were up-regulated when transfecting with miR-30a-5p inhibitors because these genes were up-regulated with upregulation of Runx2. The mRNA expression level of Runx 2 were downregulated with miR-30a-5p mimics, while upregulated with miR-30a-5p inhibitors. We also found that si-Runx2 inhibited osteogenesis and promoted inflammation, and miR-30a-5p inhibitor plus si-Runx 2 could combat the inhibitory osteogenic differentiation and promoting inflammation of si-Runx2.

We must admit that lack of clinical data is a limitation for our research. And we have also cited a paper which pointed out that miR-30a-5p have higher expression level in periodontitis patients by microarray assay [13]. More detail validation methods need to be carried out in follow-up experiments. All dates in our study suggested that miR-30a-5p inhibitor would promote osteogenesis and relieve inflammation by targeting Runx2.

\footnotetext{
(See figure on next page.)

Fig. 3 miR-30a-5p induced inflammation and inhibited osteogenesis. A The expression of miR-30a-5p was examined by qPCR in MC3T3-E1 cells after $24 \mathrm{~h}$ after transfected with relative controls (NC), miR-30a-5p mimics (mimic) and control. B The expression of miR-30a-5p was examined by qPCR in MC3T3-E1 cells after $24 \mathrm{~h}$ after transfected with relative controls (NC), miR-30a-5p inhibitors (inhibitors) and control. C The expression of IL 1-R1, IL1-R2 and TNFR1 were examined by qPCR in MC3T3-E1 cells after $24 \mathrm{~h}$ after transfected with relative controls (NC), miR-30a-5p mimics (mimic) and control. D The expression of IL1-R1, IL1-R2 and TNFR1 were examined by qPCR in MC3T3-E1 cells after 24 h after transfected with relative controls (NC), miR-30a-5p inhibitor and control. E The level of ALP was examined by ALP staining in MC3T3-E1 cells after 7 days after transfected with miR-30a-5p mimics, miR-30a-5p inhibitors and relative controls (control). F The level of Alizarin red was examined by ALP staining in MC3T3-E1 cells after 21 days after transfected with miR-30a-5p mimics, miR-30a-5p inhibitors and relative controls (control). G The expression of ALP, Runx2, OC and Col1 were examined by qPCR in MC3T3-E1 cells after $24 \mathrm{~h}$ after transfected with relative controls (NC), miR-30a-5p inhibitor and control. $\mathbf{H}$ The expression of ALP, Runx2, OC and Col1 were examined by qPCR in MC3T3-E1 cells after $24 \mathrm{~h}$ after transfected with relative controls (NC), miR-30a-5p mimics and control. All data are shown as mean \pm SEM. ${ }^{*} p<0.05 ;{ }^{* *} p<0.01$
} 
A

C
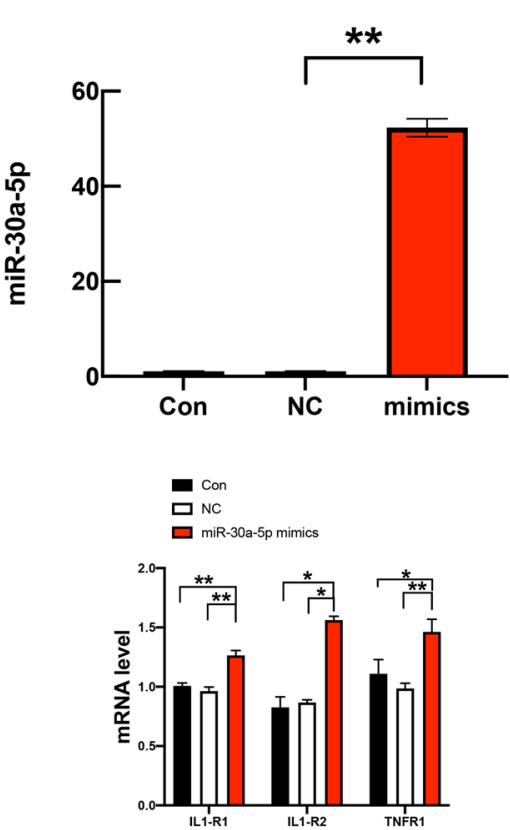

B

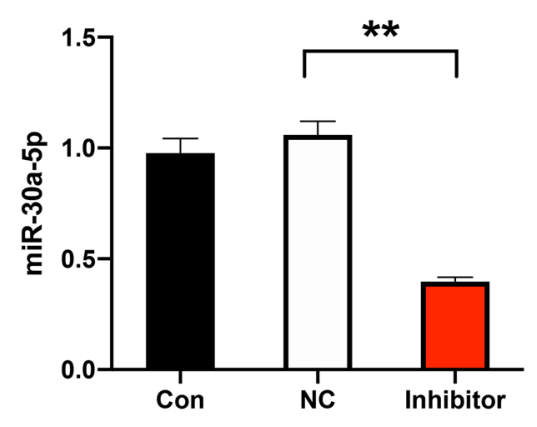

D

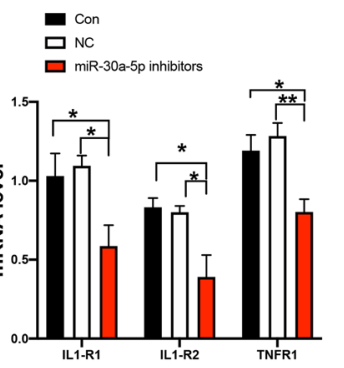

$\mathbf{E}$

Con miR-30a-5p mimics miR-30a-5p inhibitor
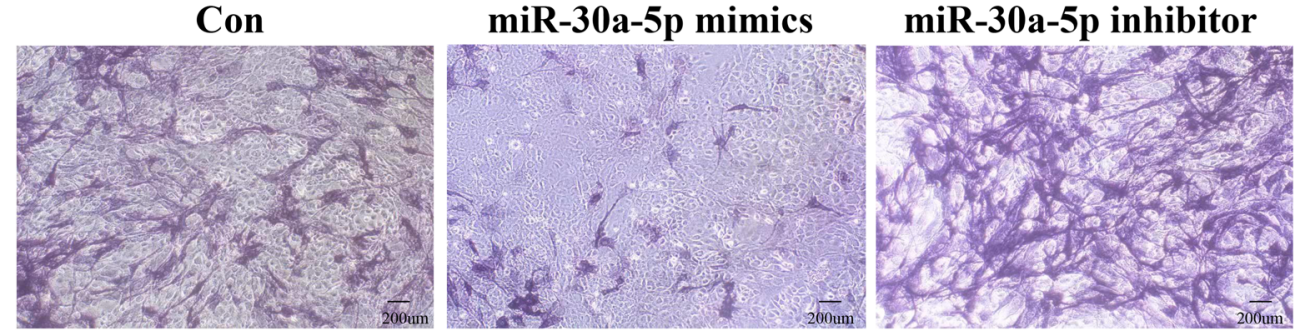

F
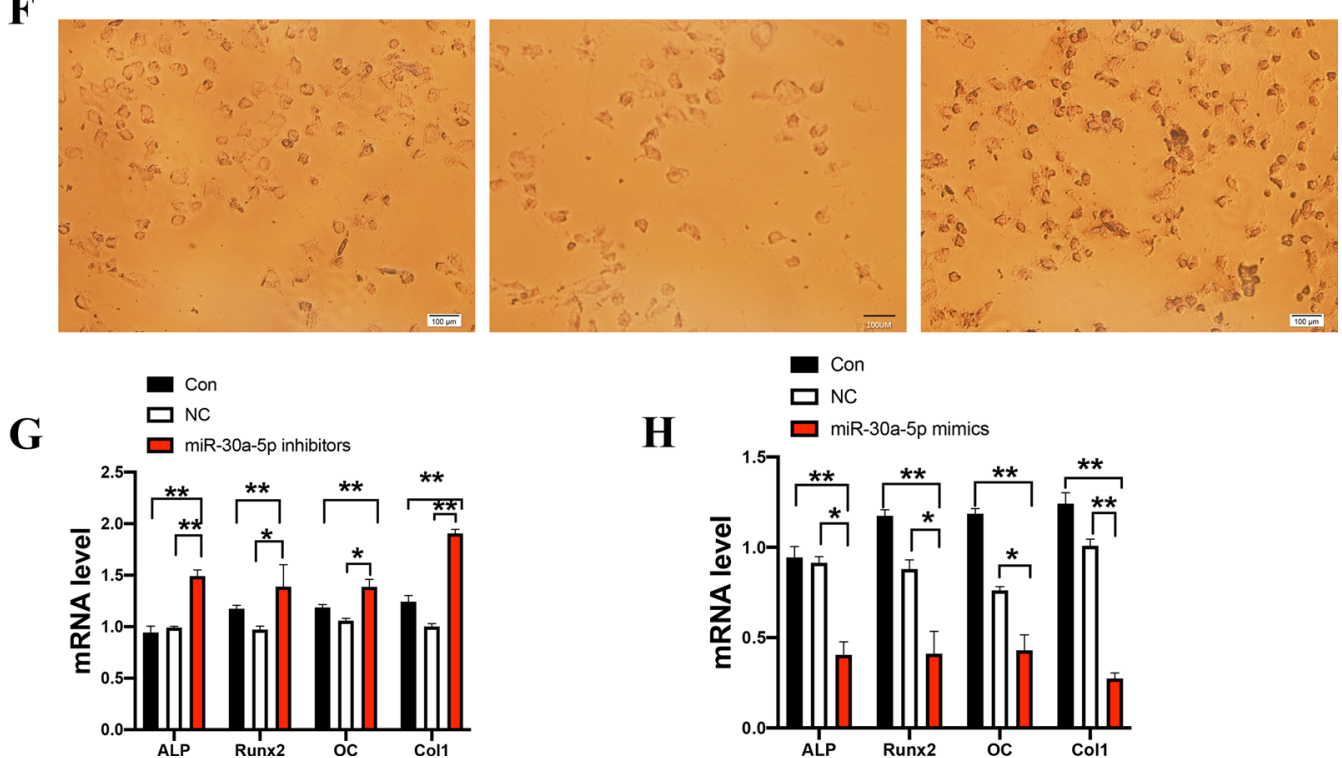

Fig. 3 (See legend on previous page.) 
A

Runx2 mRNA

5'UTR

3'UTR

miR-30a-5p

3' GAAGGUCAGUCCUACAAAUGU 5'

Runx2 3'UTR-wild

5' UGUGUGUUUACUUCAUGUUUACA 3'

Runx2 3'UTR-mutant

5' UGUGUGUUUACUUCAACAAATGT 3'

B

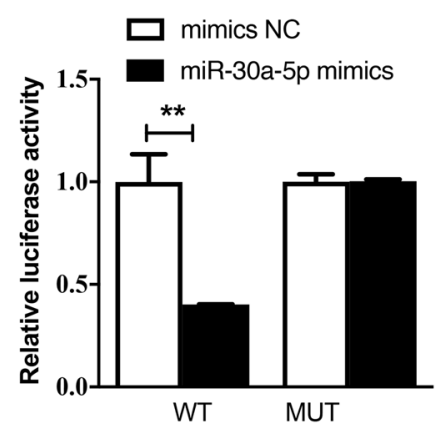

D

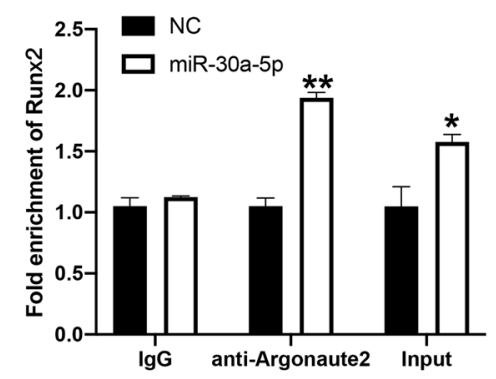

C

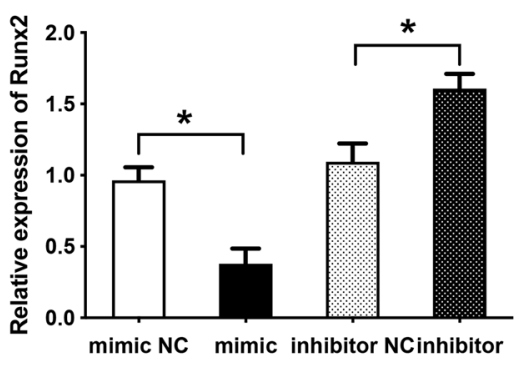

$\mathbf{E}$

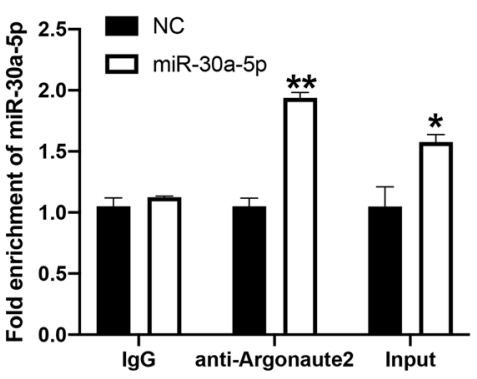

F

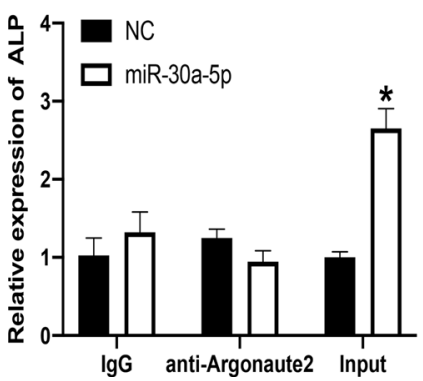

H

G

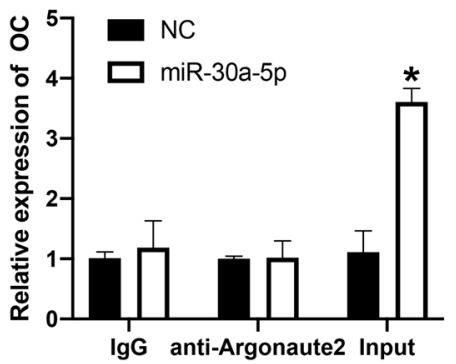

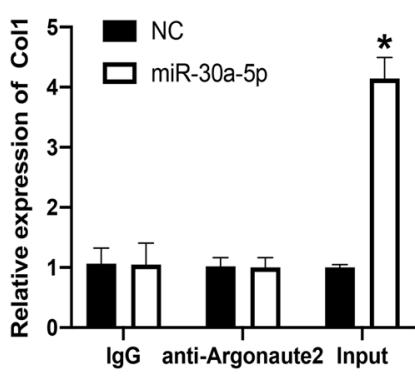

Fig. 4 miR-30a-5p directly targets Runx2 in MC3T3-E1 cell line. A The sequences of miR-30a-5p binding site within the 3'UTR of Runx2 mRNA predicted by TargetScan, and the constructs of pmir-GLO-Runx2-WT luciferase reporter and pmir-GLO-Runx2-MUT control. B The relative luciferase activity in 293T cells after $24 \mathrm{~h}$ co-transfected with miR-30a-5p mimic (miR-30a-5p) or miR-NC and WT or MUT luciferase reporter was determined by dual-luciferase reporter assays. C qRT-PCR measurement of the Runx2 mRNA expressions after $24 \mathrm{~h}$ in MC3T3-E1 cells transfected with miR-30a-5p mimic (mimic) or miR-NC and miR-30a-5p inhibitor (inhibitor) or inhibitor NC. D, E RIP assay confirmed the binding between Runx2 and miR-30a-5p in MC3T3-E1 cell line. F RIP assay confirmed that there exists no binding between ALP miR-30a-5p in MC3T3-E1 cell line. G RIP assay confirmed that there exists no binding between OC between miR-30a-5p in MC3T3-E1 cell line. H RIP assay confirmed that there exists no binding between Col1 between miR-30a-5p in MC3T3-E1 cell line. All data are shown as mean \pm SEM. ${ }^{*} p<0.05 ;{ }^{*} p<0.01$ 
A

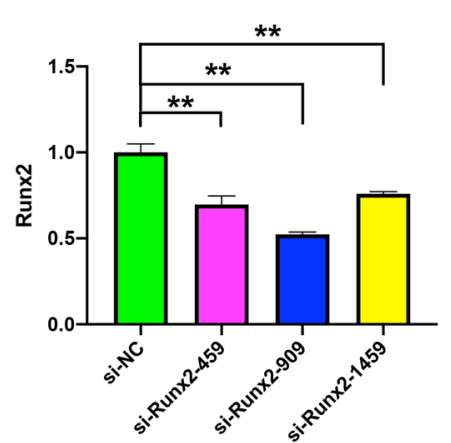

C

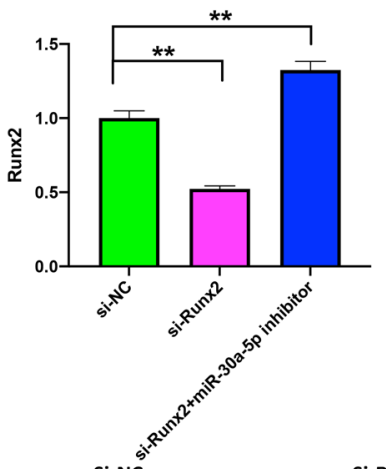

E Si-NC

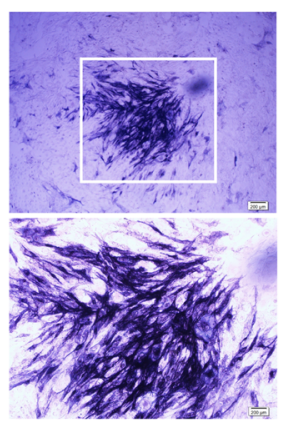

F

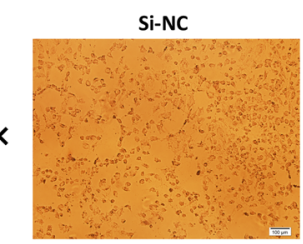

$100 \times$

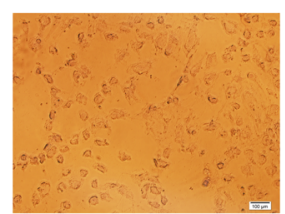

Si-Runx2
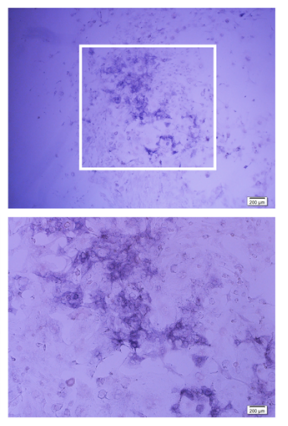

Si-Runx2
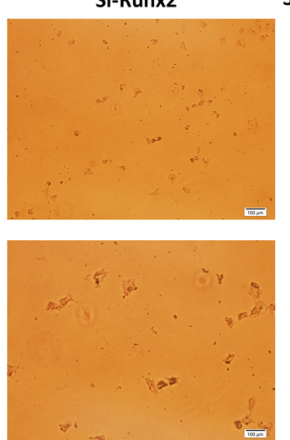

B

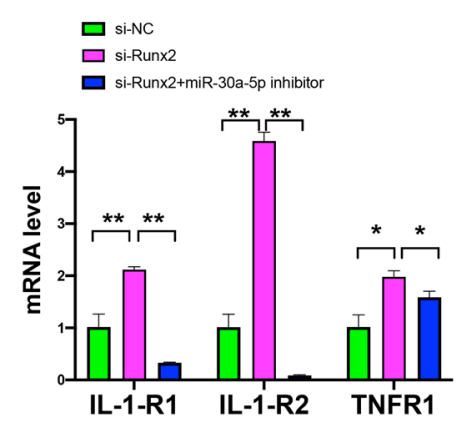

D

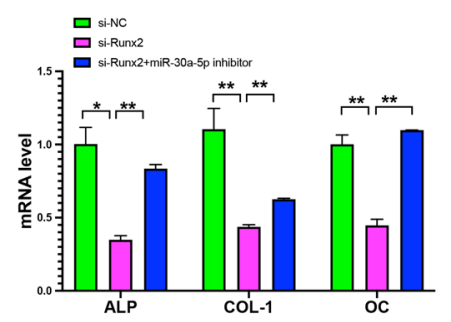

Si-Runx2+miR-30a-5p inhibitors
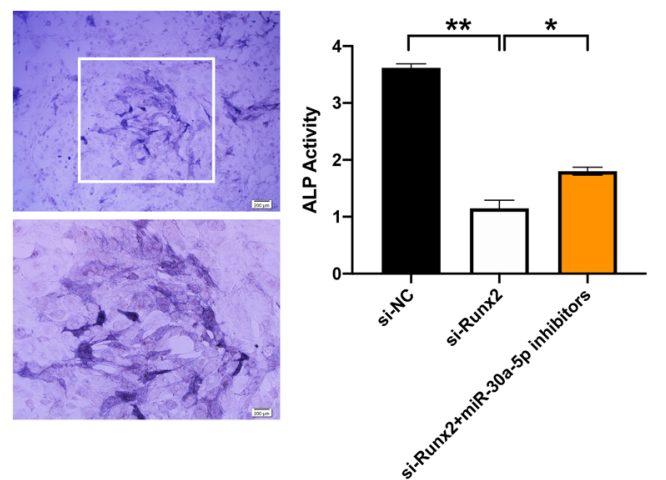

Si-Runx2+miR-30a-5p inhibitors
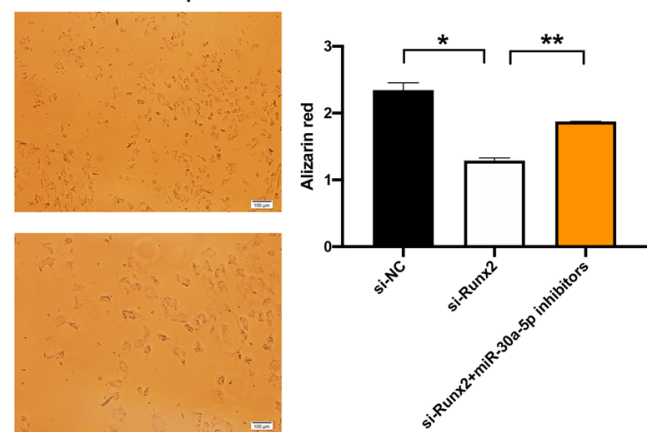

Fig. 5 si-Runx2 plus miR-30a-5p inhibitors combatted the efficiency of osteogenesis and inflammation from si-Runx2. A The expression of Runx2 was examined by qPCR in MC3T3-E1 cells after $24 \mathrm{~h}$ after transfected with three si-Runx2 (si-Runx2-459, si-Runx2-909, si-Runx2-1459) and relative controls (si-NC). B The expression of Runx2 was examined by qPCR in MC3T3-E1 cells after $24 \mathrm{~h}$ after transfected with si-Runx2, relative controls (si-NC) and si-Runx2+ miR-30a inhibitors. C The expression of ALP, COL-1 and OC were examined by qPCR in MC3T3-E1 cells after $24 \mathrm{~h}$ after transfected with si-Runx2, relative controls (si-NC) and si-Runx2 + miR-30a inhibitors. D The expression of IL-1-R1, IL1-R2 and TNFR were examined by qPCR in MC3T3-E1 cells after $24 \mathrm{~h}$ after transfected with si-Runx2, relative controls (si-NC) and si-Runx2 + miR-30a inhibitors. E The level of ALP was examined by ALP staining in MC3T3-E1 cells after 7 days after transfected with si-Runx2, relative controls (si-NC) and si-Runx2 + miR-30a inhibitors. F The level of Alizarin red was examined by Alizarin red staining in MC3T3-E1 cells after 21 days after transfected with si-Runx2, relative controls (si-NC) and si-Runx2+ miR-30a inhibitors. All data are shown as mean \pm SEM. ${ }^{*} p<0.05 ; * * 0.01$ 


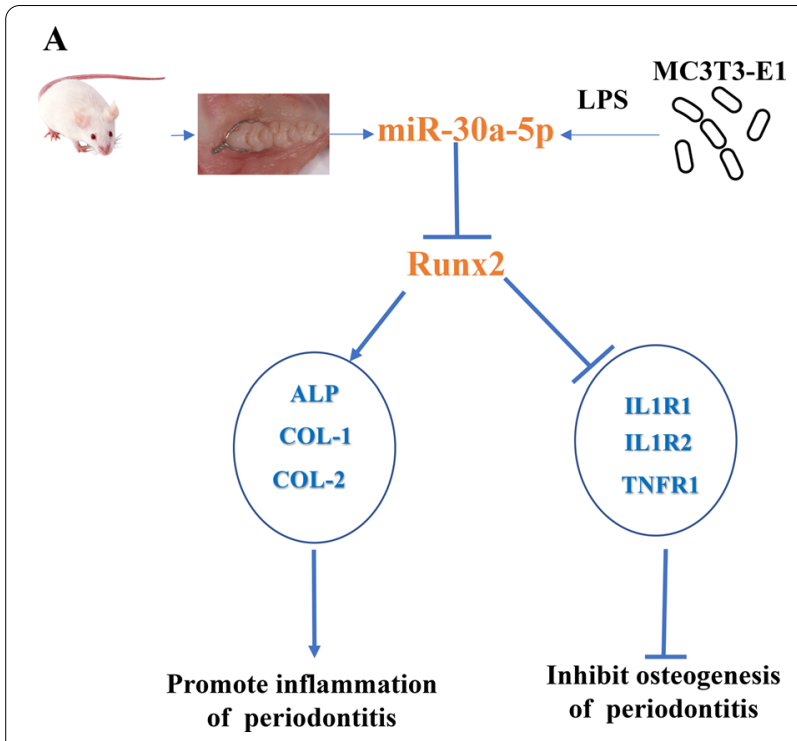

Fig. 6 A diagram of the osteogenesis and inflammation functions of miR-30a-5p inhibited osteogenesis and promoted inflammation by targeting Runx2

\section{Conclusions}

In conclusion, miR-30a-5p was upregulated in inflammatory tissue of periodontitis rat model and MC3T3E1 cell line, miR-30a-5p inhibitorS would promote osteogenesis and relieve inflammation by targeting Runx2 in MC3T3-E1 cell line under LPS-induing, which were summarized in Fig. 6. All suggested that miR-30a-5p involved in regulatory role of osteogenesis and inflammation targeting by Runx 2 in periodontitis. Our study offered a potential means for promoting bone formation and provided a novel understanding of mechanism of periodontitis.

\section{Abbreviation}

miRNA: MicroRNA

\section{Supplementary Information}

The online version contains supplementary material available at https://doi. org/10.1186/s12903-021-01882-9.

Additional file 1. Figure S1. Runx2-WT-miR-30a-5p and Runx2-MUT-miR30a-5p were cloned successfully. (A) A diagram of vector of GP-miRGLO for luciferase reporter gene experiment. (B) Sequencing of Runx2-WT-miR30a-5p and Runx2-MUT-miR-30a-5p.

\section{Acknowledgements}

Not applicable.

\section{Authors' contributions}

$X L, B Y$, and $Y Z$ designed this experiment and drafted the manuscript. $X G$ conducted the experiment. QY and QB, XL revised the draft critically. QL revised the manuscript critically. All authors approved the final manuscript.

\section{Funding}

This work was supported by the National Natural Science Foundation (Nos. $31271030,81772937,30870636)$. The funders had no role in design, collection and analysis of study, interpretation of data, or preparation of the manuscript.

\section{Availability of data and materials}

The datasets used and/or analyzed during the current study are available from the corresponding author on reasonable request.

\section{Declarations}

\section{Ethics approval and consent to participate}

All experiments performed in this study were confirmed by the Ethical Committee of the School of Stomatology, The Fourth Military Medical University (Xi'an, China, NO: kq-013) and in compliance with ARRIVE guidelines (https:// arriveguidelines.org). All methods were accordance with relevant guidelines including the revised Animals (Scientific Procedures) Act 1986 in the UK and Directive 2010/63/EU in Europe. All lab work and methods were performed by the authors of this paper.

\section{Consent for publication}

Not applicable.

\section{Competing interests}

The authors declare no competing financial interests.

\section{Author details}

${ }^{1}$ State Key Laboratory of Military Stomatology and National Clinical Research Center for Oral Diseases and Shaanxi Engineering Research Center for Dental Materials and Advanced Manufacture, Department of Implant Dentistry, School of Stomatology, The Fourth Military Medical University, Xi'an 710032, China. ${ }^{2}$ State Key Laboratory of Military Stomatology and National Clinical Research Center for Oral Diseases and Shaanxi Key Laboratory of Oral Diseases, Department of Operative Dentistry and Endodontics, School of Stomatology, The Fourth Military Medical University, Xi'an, China. ${ }^{3}$ Shanxi Provincial People's Hospital, Shanxi, China. ${ }^{4}$ Baoding First Central Hospital, Baoding, China. ${ }^{5}$ The Fourth Affiliated Hospital of Harbin Medical University, Harbin, China.

Received: 14 July 2021 Accepted: 29 September 2021

Published online: 11 October 2021

References

1. Slots J. Periodontitis: facts, fallacies and the future. Periodontol. 2017;2000(75):7-23.

2. Daiya S, Sharma RK, Tewari S, Narula SC, Kumar Sehgal P. Micronutrients and superoxide dismutase in postmenopausal women with chronic periodontitis: a pilot interventional study. J Periodontal Implant Sci. 2014:44:207-13.

3. Kinane DF, Stathopoulou PG, Papapanou PN. Periodontal diseases. Nat Rev Dis Primers. 2017;3:17038.

4. Marchesan J, Girnary MS, Jing L, Miao MZ, Zhang S, Sun L, Morelli T, Schoenfisch MH, Inohara N, Offenbacher S, Jiao Y. An experimental murine model to study periodontitis. Nat Protoc. 2018;13:2247-67.

5. van Palenstein Helderman WH, Joarder MA, Begum A. Prevalence and severity of periodontal diseases and dental caries in Bangladesh. Int Dent J. 1996:46:76-81.

6. Preshaw PM, Alba AL, Herrera D, Jepsen S, Konstantinidis A, Makrilakis K, Taylor R. Periodontitis and diabetes: a two-way relationship. Diabetologia. 2012;55:21-31.

7. Basha S, Shivalinga Swamy H, Noor Mohamed R. Maternal periodontitis as a possible risk factor for preterm birth and low birth weight-a prospective study. Oral Health Prev Dent. 2015;13:537-44.

8. Sanz M, Marco Del Castillo A, Jepsen S, Gonzalez-Juanatey JR, D'Aiuto F, Bouchard P, Chapple I, Dietrich T, Gotsman I, Graziani F, Herrera D, Loos B, Madianos P, Michel JB, Perel P, Pieske B, Shapira L, Shechter M, Tonetti M, Vlachopoulos C, Wimmer G. Periodontitis and cardiovascular diseases: consensus report. J Clin Periodontol. 2020;47:268-88. 
9. Bale BF, Doneen AL, Vigerust DJ. High-risk periodontal pathogens contribute to the pathogenesis of atherosclerosis. Postgrad Med J. 2017:93:215-20.

10. Lu TX, Rothenberg ME. MicroRNA. J Allergy Clin Immunol. 2018;141:1202-7.

11. Tiwari A, Mukherjee B, Dixit M. MicroRNA key to angiogenesis regulation: MiRNA biology and therapy. Curr Cancer Drug Targets. 2018;18:266-77.

12. Tomofuji T, Yoneda T, Machida T, Ekuni D, Azuma T, Kataoka K, Maruyama T, Morita M. MicroRNAs as serum biomarkers for periodontitis. J Clin Periodontol. 2016;43:418-25.

13. Lee $\mathrm{YH}$, Na HS, Jeong SY, Jeong SH, Park HR, Chung J. Comparison of inflammatory microRNA expression in healthy and periodontitis tissues. Biocell. 2011;35:43-9.

14. Luo Y, Peng X, Duan D, Liu C, Xu X, Zhou X. Epigenetic regulations in the pathogenesis of periodontitis. Curr Stem Cell Res Ther. 2018;13:144-50.

15. Marques-Rocha JL, Samblas M, Milagro Fl, Bressan J, Martinez JA, Marti A. Noncoding RNAs, cytokines, and inflammation-related diseases. FASEB J. 2015;29:3595-611.

16. Zhou W, Su L, Duan X, Chen X, Hays A, Upadhyayula S, Shivde J, Wang H, Li Y, Huang D, Liang S. MicroRNA-21 down-regulates inflammation and inhibits periodontitis. Mol Immunol. 2018;101:608-14.

17. Zheng Y, Dong C, Yang J, Jin Y, Zheng W, Zhou Q, Liang Y, Bao L, Feng G, Ji J, Feng X, Gu Z. Exosomal microRNA-155-5p from PDLSCs regulated Th17/Treg balance by targeting sirtuin-1 in chronic periodontitis. J Cell Physiol. 2019;234:20662-74.

18. Pedersen NJ, Jensen DH, Lelkaitis G, Kiss K, Charabi BW, Ullum H, Specht L, Schmidt AY, Nielsen FC, von Buchwald C. MicroRNA-based classifiers for diagnosis of oral cavity squamous cell carcinoma in tissue and plasma. Oral Oncol. 2018;83:46-52.

19. Vimalraj S, Arumugam B, Miranda PJ, Selvamurugan N. Runx2: structure, function, and phosphorylation in osteoblast differentiation. Int J Biol Macromol. 2015;78:202-8.

20. Komori T, Yagi H, Nomura S, Yamaguchi A, Sasaki K, Deguchi K, Shimizu Y, Bronson RT, Gao YH, Inada M, Sato M, Okamoto R, Kitamura Y, Yoshiki $\mathrm{S}$, Kishimoto T. Targeted disruption of Cbfa1 results in a complete lack of bone formation owing to maturational arrest of osteoblasts. Cell. 1997;89:755-64.

21. Komori T. Runx2, an inducer of osteoblast and chondrocyte differentiation. Histochem Cell Biol. 2018;149:313-23.

22. Enomoto H, Shiojiri S, Hoshi K, Furuichi T, Fukuyama R, Yoshida CA, Kanatani N, Nakamura R, Mizuno A, Zanma A, Yano K, Yasuda H, Higashio K, Takada K, Komori T. Induction of osteoclast differentiation by Runx2 through receptor activator of nuclear factor-kappa B ligand (RANKL) and osteoprotegerin regulation and partial rescue of osteoclastogenesis in Runx2 ${ }^{-1-}$ mice by RANKL transgene. J Biol Chem. 2003;278:23971-7.

23. Catheline SE, Hoak D, Chang M, Ketz JP, Hilton MJ, Zuscik MJ, Jonason $J H$. Chondrocyte-specific RUNX2 overexpression accelerates posttraumatic osteoarthritis progression in adult mice. J Bone Miner Res. 2019:34:1676-89.

24. Hu J, Zou WZ, Li L, Shi ZS, Liu XZ, Cai HT, Yang AF, Sun DM, Xu LL, Yang Y, $\mathrm{Li} Z \mathrm{ZH}$. Overexpressing Runx2 of BMSCs improves the repairment of knee cartilage defects. Curr Gene Ther. 2020;20:395-404.
25. Kuchler EC, Hannegraf ND, Lara RM, Reis CLB, Oliveira DSB, Mazzi-Chaves JF, Ribeiro Andrades KM, Lima LF, Salles AG, Antunes LAA, Sousa-Neto MD, Antunes LS, Baratto-Filho F. Investigation of genetic polymorphisms in BMP2, BMP4, SMAD6, and RUNX2 and persistent apical periodontitis. J Endod. 2021:47:278-85.

26. Papapanou PN. The prevalence of periodontitis in the US: forget what you were told. J Dent Res. 2012;91:907-8.

27. Huang Y. The novel regulatory role of IncRNA-miRNA-mRNA axis in cardiovascular diseases. J Cell Mol Med. 2018;22:5768-75.

28. Cao B, Zhou X, Ma J, Zhou W, Yang W, Fan D, Hong L. Role of MiRNAs in inflammatory bowel disease. Dig Dis Sci. 2017;62:1426-38.

29. Wang ZF, Hu R, Pang JM, Zhang GZ, Yan W, Li ZN. Serum long noncoding RNA LRB1 as a potential biomarker for predicting the diagnosis and prognosis of human hepatocellular carcinoma. Oncol Lett. 2018;16:1593-601.

30. Sun Y, Yang B, Lin M, Yu H, Chen H, Zhang Z. Identification of serum miR$30 a-5 p$ as a diagnostic and prognostic biomarker in colorectal cancer. Cancer Biomark. 2019;24:299-305

31. Zhu J, Zeng Y, Li W, Qin H, Lei Z, Shen D, Gu D, Huang JA, Liu Z. CD73/ NT5E is a target of miR-30a-5p and plays an important role in the pathogenesis of non-small cell lung cancer. Mol Cancer. 2017;16:34.

32. Zhao CY, Wang W, Yao HC, Wang X. SOCS3 Is Upregulated and targeted by miR30a-5p in allergic rhinitis. Int Arch Allergy Immunol. 2018;175:209-19.

33. Shangxun Z, Junjie L, Wei Z, Yutong W, Wenyuan J, Shanshou L, Yanjun W, Qianmei W, Zhusheng F, Chaoping Y, Ran Z, Wen Y, Yang H. ADAR1 alleviates inflammation in a murine sepsis model via the ADAR1-miR-30aSOCS3 axis. Mediators Inflamm. 2020;2020:9607535.

34. Correia de Sousa M, Gjorgjieva M, Dolicka D, Sobolewski C, Foti M. Deciphering miRNAs'action through miRNA editing. Int J Mol Sci. 2019;20:6249.

35. Bruderer M, Richards RG, Alini M, Stoddart MJ. Role and regulation of RUNX2 in osteogenesis. Eur Cell Mater. 2014;28:269-86.

36. Komori T. Regulation of proliferation, differentiation and functions of osteoblasts by Runx2. Int J Mol Sci. 2019;20:1694.

37. Almalki SG, Agrawal DK. Key transcription factors in the differentiation of mesenchymal stem cells. Differentiation. 2016;92:41-51.

38. Meyer MB, Benkusky NA, Sen B, Rubin J, Pike JW. Epigenetic plasticity drives adipogenic and osteogenic differentiation of marrow-derived mesenchymal stem cells. J Biol Chem. 2016;291:17829-47.

39. Li P, Yao Y, Ma Y, Chen Y. MiR-30a-5p ameliorates LPS-induced inflammatory injury in human A549 cells and mice via targeting RUNX2. Innate Immun. 2021;27:41-9.

40. Keene JD, Komisarow JM, Friedersdorf MB. RIP-Chip: the isolation and identification of mRNAs, microRNAs and protein components of ribonucleoprotein complexes from cell extracts. Nat Protoc. 2006;1:302-7.

\section{Publisher's Note}

Springer Nature remains neutral with regard to jurisdictional claims in published maps and institutional affiliations.

Ready to submit your research? Choose BMC and benefit from

- fast, convenient online submission

- thorough peer review by experienced researchers in your field

- rapid publication on acceptance

- support for research data, including large and complex data types

- gold Open Access which fosters wider collaboration and increased citations

- maximum visibility for your research: over 100M website views per year

At BMC, research is always in progress.

Learn more biomedcentral.com/submissions 